\title{
APRENDIZAGEM ATIVA PARA UMA FORMAÇÃO POR COMPETENCIAS
}

Alessandro Mattedi - alessandro.mattedi@ufes.br

Tiara Rodrigues Smarssaro de Freitas - tiara.freitas@ufes.br

Roberta Limas Gomes - rgomes@inf.ufes.br

Universidade Federal do Espírito Santo, Centro Tecnológico

Av. Fernando Ferrari, 514 - Secretaria do Centro Tecnológico

29075-910 - Campus Goiabeiras - Vitória - ES

Resumo: As metodologias de aprendizagem ativa são imprescindiveis para a formação por competências, pois permitem trabalhar de forma mais efetiva aspectos de aprendizado de conteúdos, habilidades e atitudes (CHA). No intuito de promover uma formação adequada dos estudantes, conceitos e experiências têm sido debatidos e apresentados na literatura. Buscando apresentar de forma objetiva os aspectos que envolvem o desenvolvimento das competências almejadas, este artigo traz uma síntese dos principais métodos de aprendizagem ativa que têm sido empregados nas escolas de engenharias. São também apresentados os pressupostos teóricos que embasam as metodologias, bem como quais objetivos pedagógicos são alcançados. Um estudo de caso aplicado à disciplina de Análise e Modelagem de Sistemas Dinâmicos seguindo uma abordagem de Sala de Aula Invertida é apresentado.

Palavras-chave: Formação por competências. Metodologias ativas. Metodologias de aprendizagem ativa. Educação em Engenharia.

\section{INTRODUÇÃO}

Inicialmente, é preciso compreender o que significa formar por competências. Segundo MACHADO (2012), competência está associada a pessoa, âmbito e mobilização; ou seja, uma pessoa competente é alguém integrada a uma equipe que tem capacidade de mobilizar conhecimentos disciplinares em determinado âmbito, com capacidade de extrapolação, para realizar aquilo que se deseja ou que se projeta.

Em linhas gerais o que significa um estudante ser competente? O que se deve buscar na formação por competências? Primeiro, o estudante precisa ter a capacidade de se expressar e de compreender o mundo. Segundo, o estudante precisa saber analisar os conhecimentos disciplinares, bem como ser capaz de sintetizar, decidir. Por fim, precisa ser capaz de contextualizar aquilo que se aprende, bem como extrapolar para criar o novo, para inovar (mobilização do facto para o ficto) (MACHADO, 2012). E, dentro dessas definições, é importante notar que o processo formativo por competência se dá por um conjunto de aprendizados de Conteúdos, Habilidades e Atitudes (CHA).

Outro olhar vem da neurociência que evidencia a importância do desenvolvimento de funções executivas, que garante ao estudante uma formação para ter autonomia e iniciativa própria, com propostas de objetivos de longo prazo, em que sua inteligência intrapessoal seja 
marcada de maturidade, sabedoria e criatividade no processo de auto expressão. Além disso, é primordial que os estudantes sejam conscientes e tolerantes com o processo de transformação pessoal e, portanto, capazes de um crescimento contínuo (COSENZA e GUERRA, 2011).

Nessa persperctiva, este trabalho visa apresentar um panorama de diferentes áreas de conhecimento que apontem para uma formação por competências, bem como sintetizar como as metodologias de aprendizagem ativa podem ser empregadas para essa formação. Sendo assim, um estudo de caso é apresentado de aplicação de uma metodologia de aprendizagem ativa para uma disciplina da Área de Controle de Sistemas (OGATA, 2008; KUO e GOLNARAGHI, 2003).

\section{AS BASES DAS METODOLOGIAS DE APRENDIZAGEM ATIVA}

$\mathrm{Na}$ literatura, diversos conceitos são apresentados que fundamentam as metodologias de aprendizagem ativa, aprendizagem significativa e contextualizada, tais como: formação por competências, teorias pedagógicas, epistemológicas e psicológicas (OLIVEIRA, 2019). Neste contexto, na Figura 1, apresentamos um quadro geral que interrelaciona as principais teorias e estratégias para uma efetiva formação por competências:

Figura 1 - Panorama de teorias e estratégias para uma formação por competências.

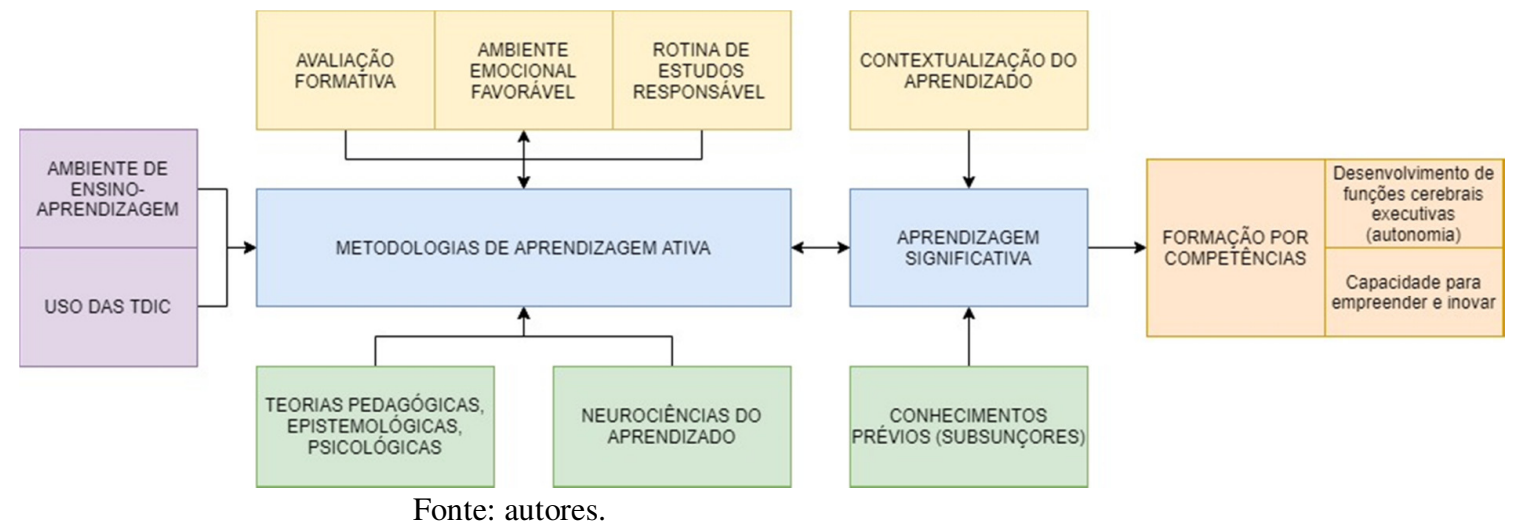

Do ponto de vista do processo formativo por competências, é importante evidenciar as metodologias de aprendizagem ativa como meios para formação acadêmica/profissional significativa e contextualizada. Na verdade, são estratégias e métodos que, fundamentadas em teorias epistemológicas, pedagógicas, psicológicas e neurocientíficas, promovem um aprendizado não arbitrário, substantivo e centrado no estudante (OLIVEIRA, 2019). Essa aprendizagem deverá estar ligada inteiramente aos conhecimentos prévios para que o processo formativo cognitivo seja bem consolidado, além do fato de que precisam visionar a importância da matéria em estudo, pois assim o aprendizado terá melhores condições de ser consolidado. Em diversos casos, é preciso ver o problema em diferentes nuances para que o aprendizado seja bem elaborado e duradouro no processo formativo neurobiológico (COSENZA e GUERRA, 2011).

Importante notar que não basta apenas "passar conteúdos" para os estudantes: é preciso que o educando seja capaz de mobilizar os conhecimentos adquiridos para conceber, projetar e/ou implementar algo em determinado âmbito, sabendo trabalhar em equipe com inteligência emocional e prática. Ou seja, o processo formativo precisa culminar no desenvolvimento de funções executivas do estudante (COSENZA e GUERRA, 2011) para uma formação por competências para inovar e empreender dentro de um contexto social, empresarial e ambiental. 
Também é mister realçar que essas metodologias precisam estar embasadas em alguns pressupostos teóricos, que são listados brevemente a seguir.

I) Teoria construtivista: o aprendizado ocorre quando o sujeito age sobre os objetos em estudo e sofre influências desta ação sobre si mesmo; essa ação precisa ser orientada por meio de questionamentos que produzam desequilíbrios e reflexões, alocando ao professor papel de mediador para levar o estudante às principais questões do estudo (PIAGET, 2007). Importante também ressaltar que de certa forma a concepção das metodologias ativas é relativamente antiga e surge com o movimento da Escola Nova de John Dewey, que defendia uma metodologia de ensino pautada pelo "aprender fazendo" (learning by doing), centrada na aprendizagem pela experiência e no desenvolvimento da autonomia do aprendiz (BACICH e MORAN, 2018).

II) Teoria dialógica, participativa e conscientizadora de Paulo Freire (FREIRE, 2001): está relacionada com a teoria de Piaget, que aponta o diálogo como fenômeno humano, cujos elementos constitutivos comportam a ação e a reflexão e, dessa forma, pode gerar aprendizado.

III) Teoria psicológica de AUSUBEL (AUSUBEL, NOVAK e HANESIAN 1980; AUSUBEL, 2003): destaca o aspecto motivacional relacionado à aprendizagem significativa $\mathrm{e}$ contextualizada.

IV) Neurociências do aprendizado (COSENZA e GUERRA, 2011): explica a importância do processo de ensino-aprendizagem do ponto de vista neurobiológico e assim fundamenta a proposição de metodologias mais efetivas de aprendizado.

Para a operacionalização das metodologias de aprendizagem ativa, cabe destacar a importância dos ambientes de aprendizagem que devem substituir as tradicionais "salas de aula". Para isso, é necessário haver readaptação desses ambientes para que os trabalhos de aprendizagem sejam realizados de uma forma mais cooperativa e colaborativa. Também importante sublinhar a importância dos recursos de Tecnologias Digitais de Informação e Comunicação (TDIC) para que os encontros presenciais com o professor sejam mormente para significar o aprendizado dos estudantes. Isto, principalmente, por meio de discussão de exercícios envolvendo conceitos fundamentais, problemas e projetos contextualizados e experimentos laboratoriais e computacionais.

O ambiente emocional deve ser o melhor possível, de modo que haja sempre uma postura curiosa, aberta, alegre, crítica, reflexiva, comprometida, fraterna, ética e, por fim, com disposição para aceitar desafios (OLIVEIRA, 2019), em que o estudante encontre uma rotina de estudos saudável e responsável. Aqui é importante destacar que o sucesso das novas metodologias de aprendizagem depende fundamentalmente do comprometimento dos estudantes, do protagonismo dos mesmos em assumir com responsabilidade suas tarefas, de entender que a responsabilidade pelo aprendizado deve ser compartilhada no decorrer do processo de ensino-aprendizagem. Dessa forma, haverá um ambiente saudável, proveitoso e responsável.

No tocante ao processo avaliativo, importante frisar que precisa ser contínuo e focado no aprendizado do estudante, no sentido de apontar as deficiências que precisam ser corrigidas, referente ao aprendizado de conteúdos, habilidades e atitudes, em consonância com a proposta curricular de formação por competências. Para tanto, importante empregar a classificação de aprendizado de acordo com a Taxionomia do Domínio Cognitivo de Bloom (Bloom et. al., 1956) para determinar os seis níveis de aprendizado desejados do ponto de vista cognitivo: conhecimento, compreensão, aplicação, análise, síntese (implementação) e avaliação (ELMÔR FILHO et. al., 2019). Para que o aprendizado possa ser galgado em níveis crescentes de complexidade para alcançar uma formação mais efetiva. 
Por fim, importante realçar o papel do professor, que deve ser exercido muito mais como mediador e motivador. É preciso dizer que o professor precisa tornar o momento de aula muito mais interessante e significativo do ponto de vista do aprendizado. Os estudantes precisam chegar no momento de aula muito mais preparados, de modo que o aprendizado possa ser aprofundado de acordo com o nível proposto de Bloom, por meio de resolução de problemas conceituais, projetos contextualizados, entre outros, de modo que o ambiente seja repleto de curiosidade, entusiasmo, envolvimento e desafios, para uma formação de engenheiros muito mais criativos. Por isso, o papel do professor na verdade ganha um outro destaque, quando se considera essa meta de ideal de ensino, sendo que somente com muita dedicação e humildade por parte do professor, em se render que é preciso ele mesmo angariar novas competências para sua carreira docente. Ou seja, a mudança de paradigma de ensino é um desafio que se impõe ao docente. Não basta ensinar como a ele foi ensinado: é fundamental perceber que o mundo mudou, os alunos têm um perfil diferente, e para propiciar ao estudante alcançar uma aprendizagem real, o processo de ensino precisa ser reinventado, sempre objetivando fazer com que o educando se torne competente naquilo que a profissão almejada requer que ele seja.

\subsection{Síntese das metodologias de aprendizagem ativa}

Muitas são as metodologias de aprendizagem ativa que podem ser aplicadas na Educação em Engenharia, presentes em diversas referências de educação (ELMÔR et. al., 2019; OLIVEIRA, 2019; BACICH e MORAN, 2018; MAZUR, 1997). A seguir são listadas as principais que têm sido empregadas na Engenharia:

- Sala de aula invertida (Flipped Classroom);

- Instrução por pares (Peer Instruction);

- Aprendizagem baseada em problemas (PBL);

- Aprendizagem baseada em projetos $(\mathrm{PjBL})$;

- Aprendizagem Personalizada.

Cabe comentar que a Aprendizagem Personalizada se trata de uma metodologia adaptativa de acordo com o aprendizado do estudante, em que se propõe a oferta de um ensino customizado, em que normalmente se utilizam recursos de inteligência artificial para o direcionamento das melhores trilhas de atividades pedagógicas, com o apoio de recursos de tecnologia da educação, de tal forma que se possam corrigir quaisquer deficiências de aprendizado do estudante.

Em termos gerais os objetivos principais dessas novas metodologias são basicamente tornar o momento de aula mais proveitoso, engajar o estudante no processo de aprendizado, promover um ambiente de aprendizado mais cooperativo e colaborativo e, principalmente, promover uma formação de engenheiros mais inovadores e empreendedores. Quanto ao momento de aula, frisamos que há certamente espaço para aulas expositivas de acordo com o conteúdo e disciplina envolvida.

Em suma, a Tabela 1 mostra os objetivos pedagógicos dessas novas metodologias de acordo o desenvolvimento de aprendizado de conteúdos (cognitivo), habilidades (psicomotor) e atitudes (afetivo), com a meta da formação por competências. 
"Os desafios para formar hoje o engenheiro do amanhã"

Tabela 1 - Objetivos pedagógicos visados pelas novas metodologias.

\begin{tabular}{l|l}
\hline Objetivo pedagógico & Desenvolvimento desejado \\
\hline $\begin{array}{l}\text { Alcançar uma aprendizagem } \\
\text { significativa, contextualizada e } \\
\text { duradoura; }\end{array}$ & cognitivo - conteúdos \\
\hline $\begin{array}{l}\text { Desenvolver habilidades de síntese e } \\
\text { avaliação, por meio de aprofundamento } \\
\text { problemas e/ou projetos contextualizados, } \\
\text { que empreguem recursos tecnológicos de } \\
\text { simulação, projeto e implementação; }\end{array}$ & cognitivo e psicomotor - habilidades \\
\hline $\begin{array}{l}\text { Desenvolver atitudes profissionais e } \\
\text { pessoais, principalmente em relação ao } \\
\text { trabalho cooperativo e colaborativo, à } \\
\text { ética, à responsabilidade e à liderança; }\end{array}$ & afetivo - atitudes \\
\hline $\begin{array}{l}\text { Desenvolver habilidades interpessoais de } \\
\text { comunicação escrita, oral e gráfica. }\end{array}$ & habilidades interpessoais \\
\hline
\end{tabular}

Fonte: autores.

\subsection{Estudo de Caso}

Para exemplificar, toma-se o caso da disciplina de Análise e Modelagem de Sistemas Dinâmicos, disciplina básica da Área de Controle de Sistemas dos Cursos de Engenharia Elétrica e de Engenharia de Computação do Centro Tecnológico / UFES.

Propõem-se alguns elementos fins de aprendizado para o caso:

- o estudante precisa ser capaz de modelar e representar sistemas contínuos e discretos, para saber se expressar e compreender os fenômenos físicos;

- o estudante precisa saber analisar e sintetizar sistemas de controle contínuos e discretos;

- o estudante precisa relacionar os conhecimentos disciplinares à realidade de controle $\mathrm{e}$ automação do mercado produtivo, para poder empreender e inovar soluções, tendo em vista o desenvolvimento de funções executivas; porém, é fato que o processo de formação por competências é bem mais complexo e envolve uma série de áreas, como será mostrado ao longo deste artigo.

A proposta metodológica de ensino é, em grande parte, baseada pelo emprego da Sala de Aula Invertida, em que a disciplina está dividida em cinco módulos de aprendizado (Quadro 1) (OGATA, 2008; KUO e GOLNARAGHI, 2003), sendo que processo de ensinoaprendizagem é realizado de acordo com uma rotina de atividades propostas no Quadro 2.

No tocante ao desenvolvimento de habilidades e atitudes, propõe-se:

- Trabalhar habilidades de analisar e simular sistemas usando softwares computacionais, como Matlab, Scilab, entre outros;

- Incentivar atitudes de proatividade, responsabilidade, cooperatividade, colaboratividade e liderança. 
Quadro 1 - Módulos da disciplina.

\begin{tabular}{|c|c|}
\hline $\begin{array}{l}\text { Módulo } 1 \text { - Modelagem e descrição de } \\
\text { sistemas de ordem reduzida }\end{array}$ & $\begin{array}{l}\text { - Modelagem de circuitos elétricos RLC, } \\
\text { circuitos envolvendo amplificadores } \\
\text { operacionais, motor DC com } \\
\text { engrenagens; } \\
\text { - Transformada de Laplace; } \\
\text { - Função de transferência de sistemas } \\
\text { contínuos no tempo; } \\
\text { - Diagrama de blocos; } \\
\text { - Análise dinâmica de sistema de } 2 a \\
\text { ordem. }\end{array}$ \\
\hline $\begin{array}{l}\text { Módulo } 2 \text { - Modelagem e descrição de } \\
\text { sistemas de ordem maior }\end{array}$ & $\begin{array}{l}\text { - Modelagem de sistemas mecânicos } \\
\text { translacionais e rotacionais; } \\
\text { - Representação em variáveis de estado; } \\
\text { - Diagrama de blocos de estado; } \\
\text { - Diagrama de fluxo de sinal, fórmula de } \\
\text { Mason; } \\
\text { - Análise de resposta de sistemas em } \\
\text { variáveis de estado; } \\
\text { - Modelagem e simulação numérica } \\
\text { computacional envolvendo sistemas não- } \\
\text { lineares: suspensão eletromagnética, } \\
\text { tanques acoplados, sistema de } \\
\text { aquecimento, entre outros; } \\
\text { - Linearização de sistemas. }\end{array}$ \\
\hline $\begin{array}{l}\text { Módulo } 3 \text { - Introdução a Sistemas de } \\
\text { Controle }\end{array}$ & $\begin{array}{l}\text { - Controle por realimentação: malha de } \\
\text { controle (contínua e discreta); } \\
\text { - Controle antecipatório (feedforward); } \\
\text { - Simulação numérica de controle PID; } \\
\text { - Análise de estabilidade e de erro } \\
\text { estacionário de sistemas de controle; } \\
\text { - Métodos heurísticos de sintonia do PID; } \\
\text { - Implementação de PID em controlador } \\
\text { industrial (PLC). }\end{array}$ \\
\hline $\begin{array}{l}\text { Módulo } 4 \text { - Transformada Z e Sistemas } \\
\text { Amostrados }\end{array}$ & $\begin{array}{l}\text { - Introdução a sistemas discretos no } \\
\text { tempo; } \\
\text { - Transforma Z e transformada inversa; } \\
\text { - Função de transferência discreta; } \\
\text { - Sistemas Amostrados. }\end{array}$ \\
\hline $\begin{array}{l}\text { Módulo } 5 \text { - Análise de Sistemas Discretos } \\
\text { no tempo }\end{array}$ & $\begin{array}{l}\text { - Análise em malhas de controle de } \\
\text { sistemas discretos no tempo; } \\
\text { - Mapeamento entre os planos s e z; } \\
\text { - Representação em variáveis de estado } \\
\text { de sistemas de tempo discreto. }\end{array}$ \\
\hline
\end{tabular}

Fonte: autores. 
Quadro 2 - Atividades de Ensino-Aprendizagem.

$\left.1^{\mathrm{a}}\right)$ DIAGNÓSTICO DE FALHAS DE APRENDIZADO DE CONTEÚDOS PRÉVIOS - atividade individual - remota ou presencial;

$2^{\mathrm{a}}$ ) TRILHAS DE CONTORNO - atividade individual - remota ou presencial;

$3^{\mathrm{a}}$ ) MOMENTO DE AULA EXPOSITIVA PARA DISCUSSÃO DOS CONTEÚDOS

- atividade em turma - presencial

$\left.4^{\mathrm{a}}\right)$ RESOLUÇÃO E DISCUSSÃO DE PROBLEMAS E APRESENTAÇÃO DO PROJETO - atividade em grupos de dois ou três - presencial e remota;

$5^{\mathrm{a}}$ ) AVALIAÇÃO ESCRITA DE CONTEÚDOS - atividade individual - presencial;

$6^{\text {a }) ~ P R O J E T O ~-~ a t i v i d a d e ~ e m ~ g r u p o ~-~ p a r a ~ s e r ~ i m p l e m e n t a d o ~ r e m o t a m e n t e ~ e ~}$ apresentado presencialmente;

$7^{\mathrm{a}}$ ) AVALIAÇÃO DE COMPETÊNCIAS E/OU HABILIDADES E/OU ATITUDES

POR MEIO DE PROJETO - atividade em grupo - presencial ou/e remotamente

Fonte: autores

Os materiais para atividade remota estão sendo gerados por videoaulas, webinars, notas de aulas, exercícios, problemas e projetos contextualizados de simulação computacional. Os resultados de aprendizado têm sido muito interessantes e promissores dentro dos objetivos propostos da disciplina. O estudante munido de material para estudo remoto pode realizar seu estudo de forma autônoma no tempo mais apropriado e repetir esse processo quantas vezes achar necessário, por meio de videoaulas. O momento de aula, que acontece por meio de videoconferências (durante a pandemia) torna-se mais significativo para discussões e aprofundamentos de problemas conceituais e projetos contextualizados de simulação. Também, é importante comentar que tem sido notável a discussão entre os estudantes para o compartilhamento de aprendizado, o que possibilita o desenvolvimento de atitudes de cooperação, colaboração e liderança. Desse modo, a satisfação por parte dos estudantes pelo método de aprendizagem aplicado na disciplina tem sido bem interessante e promissora.

\section{CONSIDERAÇÕES FINAIS}

Diante da urgência da grande transformação da educação em engenharia no Brasil, este trabalho apresentou um resumo das principais metodologias de aprendizagem ativa empregadas no ensino de engenharia para permitir ao estudante uma formação por competências. Dentre as técnicas apresentadas no trabalho, pode-se validar a experiência da Sala de Aula Invertida, aplicada no curso de Análise e Modelagem de Sistemas Dinâmicos. Resultados preliminares dessa estratégia de ensino podem ser medidos na participação mais efetiva dos alunos e na motivação crescente no estudo dos conteúdos abordados na disciplina, além da percepção da importância do professor como mediador e de ter o aluno como centro da aprendizagem. Tais resultados incentivam a continuidade do processo de mudança de paradigma no ensino, especialmente para os cursos de Engenharia no país, para que se possa formar engenheiros mais preparados para inovar e empreender, com capacidade para transformar a sociedade brasileira, do ponto de vista econômico, político, social e ambiental, para o bem comum de todos.

\section{REFERÊNCIAS}

AUSUBEL, D.P., NOVAK, J.D., HANESIAN, H. Psicologia Educacional, 2a ed. Rio de Janeiro: Interamericana, 1980. 
AUSUBEL, D.P., Aquisição e Retenção de Conhecimentos - Uma Perspectiva Cognitiva. Lisboa: Plátano Edições Técnicas, 2003.

COSENZA, Ramon M., GUERRA, Leonor B. Neurociência e Educação. Porto Alegre: ARTMED Editora, 2011.

ELMÔR FILHO, Gabriel. SAUER, Laurete Zanol, DE ALMEDIA, Nival Nunes, VILLASBOAS, Valquíria. Uma Nova Sala de Aula é Possível - Aprendizagem Ativa na Educação em Engenharia. Rio de Janeiro: Editora LTC. 2019.

FREIRE, Paulo. Pedagogia da Autonomia - saberes necessários à prática educativa, 18a ed. São Paulo: Paz e Terra, 2001.

KUO, Benjamim C.; GOLNARAGHI, M.F. Automatic Control Systems. 8th ed. New York, N.Y. John Wiley, 2003.

MACHADO, Nilson José. Educação por competências. Youtube, 6 fev. 2012. Disponível em <https://www.youtube.com/watch?v=TgS6VNI328U>. Acesso em: 31 jul. 2020.

MAZUR, E. Peer Instruction. Upper Saddle River, NJ: Prentice Hall, 1997.

BACICH, Lilian; MORAN, José (orgs.). Metodologias Ativas para uma aprendizagem mais profunda. Porto Alegre: Penso Editora, 2018. p. 1-25.

OGATA, K. Engenharia de Controle Moderno - 4a edição. Rio de Janeiro: LTC, 2008.

PIAGET, J. Epistemologia Genética, 3ed. São Paulo: Martins Fontes, 2007.

OLIVEIRA, Vanderli Fava (org.). A Engenharia e as Novas DCNs - Oportunidades para Formar Mais e Melhores Engenheiros. Rio de Janeiro: LTC, 2019. p. 146-181.

\section{ACTIVE LEARNING TO ACHIEVE COMPETENCY-BASED EDUCATION}

Abstract: Active learning methodologies are absolutely necessary to achieve competencybased education. They enable to effectively manage learning aspects involving knowledge, skills and attitudes. To promote appropriate education levels, concepts and experiences have been discussed in the literature. In order to objectively present the various aspects related to the development of the aimed competencies, this article provides a synthesis of the main active learning approaches that have been employed by engineering schools. The theoretical assumptions supporting those approaches and their pedagogical objectives are also presented. A pilot case study applied to the Modeling and Analysis of Dynamic Systems course based on the Flipped Classroom approach is then discussed.

Keywords: Competency-Based Education. Active Methodologies. Active Learning Methodologies. Engineering Education. 